\title{
FONOAUDIOLOGIA E ESTÉTICA FACIAL: ESTUDO DE CASOS
}

\section{Speech therapy and facial esthetic: cases studies}

\author{
Carolina Paes ${ }^{(1)}$, Paula Nunes Toledo ${ }^{(2)}$, Hilton Justino da Silva ${ }^{(3)}$
}

\begin{abstract}
RESUMO
Objetivo: caracterizar as modificações presentes no comportamento facial após tratamento fonoaudiológico proposto para face, através da comparação das medidas da projeção do sulco nasogeniano ao tragus pré e pós-tratamento fonoaudiológico Métodos: a população foi constituída de 10 professores atendidos no Hospital dos Servidores do Estado de Pernambuco de ambos os sexos, e com idade entre 33 e 63 anos. O processo de coleta de dados consistiu de protocolos de anamnese e avaliação, protocolo de desempenho, além do registro fotográfico. Foi realizada a medida da projeção do sulco nasogeniano ao tragus. Durante dezesseis sessões foram trabalhados exercícios isotônicos, isométricos, isocinéticos, massagens, manipulações faciais, equilíbrio funcional estomatognático, além de orientações aos cuidados faciais. Resultados: foi constatada uma redução das medidas da projeção do sulco nasogeniano ao tragus em ambos os lados, observando-se também um notório equilíbrio entre os lados direito e esquerdo. Conclusão: o programa de tratamento proposto para face reduziu as medidas da projeção do sulco nasogeniano ao tragus e proporcionou um equilíbrio entre essas medidas.
\end{abstract}

DESCRITORES: Fonoaudiologia; Face; Estética

\section{INTRODUÇÃO}

O fato de o fonoaudiólogo dominar os conhecimentos específicos das funções vitais como respirar, sugar, mastigar, deglutir e falar permite que o trabaIho na área da Fonoaudiologia estética transcorra de forma harmônica e equilibrada ${ }^{1}$.

É observado um estreito relacionamento entre as marcas e vincos de expressão ao redor da boca e o uso da musculatura oral, visto que rugas indesejá-

(1) Fonoaudióloga do Hospital dos Servidores do Estado de Pernambuco; Coordenadora e professora da Unidade Especializada em Voz e Face (VOCAL \& FACE); Docente do curso de Fonoaudiologia da Faculdade Integrada do Recife; Mestre em Anatomia Patológica pela Universidade Federal de Pernambuco.

(2) Fonoaudióloga; Docente do Centro Universitário das Faculdades Metropolitanas Unidas e do curso de pós-graduação do CEFAC - Saúde Educação; Mestre em Fonoaudiologia pela Pontifícia Universidade Católica de São Paulo; Doutoranda em Ciências, pela Faculdade de Medicina da Universidade de São Paulo, Área de Cirurgia Plástica.

(3) Fonoaudiólogo docente do curso de Fonoaudiologia da Faculdade Integrada do Recife e da Universidade Federal de Pernambuco; Coordenador e professor do Centro de Estudo e Pesquisa em Motricidade Orofacial; Doutor em Nutrição pela Universidade Federal de Pernambuco. veis podem ser resultantes de posturas e movimentos repetidos realizados para mastigar, deglutir, respirar e falar, bem como tais rugas podem sofrer influência da tensão exagerada dos músculos da face ${ }^{2}$.

O relacionamento da motricidade oral influindo nas questões da estética, da beleza e do rejuvenescimento, bem como na suavização das rugas de expressão facial é observado por meio do trabalho integrado entre a Fonoaudiologia e a Dermatologia, evidenciando que este trabalho em conjunto pode prevenir e diminuir a presença dessas rugas, através do reequilíbrio das funções estomatognáticas e do relaxamento da musculatura da face ${ }^{3}$.

A preocupação com os princípios da Fonoaudiologia e estética foi o motivo para a elaboração do Programa de Aprimoramento Muscular em Fonoaudiologia Estética Facial (PAMFEF), onde é preconizada a importância da harmonia anatômica e funcional ${ }^{4}$.

A eficiência dos exercícios isométricos na musculatura da mímica da face também pode ser analisada, quando utilizada em favor da estética visando diminuir a flacidez muscular, rugas e marcas de expressão. Observou-se que, a realização sistemática dos exercícios isométricos pelo período de três meses mostrou favorecer a diminuição das rugas, marcas de expressão e flacidez facial, com variação do grau e localização da melhora ${ }^{5}$. 
Cabe a Fonoaudiologia o papel de aprofundar estudos sobre o sistema neuromuscular da face e suas funções, uma vez que esta dispõe de instrumental teórico-prático de intervenção sobre o sistema muscular oral e seu desempenho funcional, sendo proposta a atuação do fonoaudiólogo no tratamento das rugas de expressão ${ }^{6}$.

Com o advento da toxina botulínica e das técnicas de preenchimento para o tratamento estético da face, surgiram mais estudos sobre as contrações musculares, podendo-se considerar mais objetivamente a importância do fonoaudiólogo nesta área, pois muitas rugas estão relacionadas à expressão facial e contração exagerada da musculatura perioral durante a realização das funções. O conhecimento do fonoaudiólogo Especialista em Motricidade Orofacial sobre diagnóstico, aperfeiçoamento e reabilitação dos aspectos estruturais e funcionais das regiões orofacial e cervical o credita para atuação nesta área, promovendo um equilíbrio funcional e estético na face ${ }^{7}$.

Além de conhecer as estruturas musculares que compõe a face e o pescoço, o fonoaudiólogo deve conhecer o tecido tegumentar, elemento que demonstra a harmonia das formas e cuja função é proteção, termo regulação, trocas metabólicas e sensibilidade e o sistema músculo aponeurótico superficial (SMAS) que tem como função amplificar as contrações dos músculos da expressão facial. A partir destes conhecimentos propõe-se uma atuação fonoaudiológica na estética da face com o objetivo de auxílio à cosmética realizando exercícios com a estrutura intra-oral, trabalho com o equilíbrio funcional estomatognático e com a conscientização da redução dos hábitos causadores das marcas de expressão facial ${ }^{8}$.

O trabalho com a estética da face, nova área de atuação dentro da Motricidade Orofacial, propõe o reequilíbrio das funções estomatognáticas, visando a uma organização miofuncional e minimização de movimentos desnecessários; relaxamento da musculatura orofacial através de massagens, alongamentos e movimentos específicos; diminuição do abuso na mímica facial, reorganização postural; promoção da suavização da fisionomia, além de dar ao rosto um aspecto mais harmonioso, rejuvenescido e descansado, tornando a pele mais tonificada, brilhante e suave ${ }^{9}$.

Os pacientes que buscam o tratamento na área de motricidade orofacial apresentam alterações que comprometem a estética e muitas vezes a auto-estima. Embora algumas vezes não se queixem dessas alterações, quando abordados na avaliação demonstram um maior interesse no tratamento. A atuação fonoaudiológica na estética da face visa prevenir e adequar as alterações dos músculos mímicos e da mastigação desencadeadas pelo envelhecimento, pelos movimentos exagerados da mímica facial ou por distúrbios orofaciais e cervicais ${ }^{10}$.
A sistematização do estudo em estética facial no âmbito da Fonoaudiologia é relativamente recente não estando disponível uma bibliografia ampla na área específica, fazendo-se necessário, além de lançar mão da interdisciplinaridade, o desenvolvimento de novos trabalhos nesta área.

Considerando que o fonoaudiólogo tem o domínio dos conhecimentos específicos de funções essenciais como respiração, sucção, mastigação, deglutição, voz e fala, e isto o credencia a um trabalho globalizado e integrado na área da estética da face, buscou-se através da terapia miofuncional e do reequilíbrio das funções estomatognáticas a criação e execução de protocolos com a finalidade de minimizar rugas de expressão facial, equilibrar o tônus da face, dando ao rosto um aspecto mais harmonioso e simétrico, favorecendo uma expressão mais equilibrada e também sinalizando para um aumento na auto-estima e melhoria na qualidade de vida.

O objetivo desta pesquisa é caracterizar as modificações presentes no comportamento facial após tratamento fonoaudiológico proposto para face, bem como analisar as medidas da projeção do sulco nasogeniano ao tragus pré e pós-tratamento fonoaudiológico.

\section{MÉTODOS}

O estudo foi desenvolvido no Ambulatório de Fonoaudiologia do Hospital dos Servidores do Estado (HSE) do Instituto de Recursos Humanos do Estado de Pernambuco (IRH-PE). A população de estudo consistiu de 10 professores de ambos os gêneros. As idades variaram de 33 a 63 anos (média de idade de 43,5 anos). Este grupo foi selecionado aleatoriamente no mês de Julho dentre o universo de 40 sujeitos atendidos em grupos para reabilitação vocal, no ano de 2003, neste setor. Ocorrendo a intervenção fonoaudiológica no período entre Agosto e Dezembro do mesmo ano, com 16 sessões, sendo 01 encontro semanal com tratamento uniforme para todos os sujeitos da pesquisa. Trata-se de um estudo analítico descritivo e comparativo longitudinal em série de casos.

Ressalta-se que mesmo sendo escolhidos aleatoriamente nenhum dos voluntários da pesquisa apresentavam histórico de cirurgia plástica, nem tão pouco foram submetidos a outros métodos de amenização e/ou suavização de rugas na face.

O processo de coleta de dados consistiu de um protocolo de anamnese, avaliação, registro fotográfico e um protocolo de desempenho ${ }^{11}$. No protocolo de anamnese foram levantados os dados relacionados à história facial, do sistema estomatognático dos pacientes, com perguntas semi-estruturadas para permitir uma possível tabulação dos dados. O protocolo de avaliação foi constituído por itens direcionados 
para avaliação da postura e tensão corporal e facial, mímica facial, mensuração da face - mensuração da projeção do sulco nasogeniano ao tragus da hemiface direita e esquerda, estruturas do sistema estomatognático e das funções estomatognáticas clássicas.

As medidas da face foram obtidas com um paquímetro marca Westernâ de fabricação Argentina, com acurácia de 0,02 mm. Para a medida da projeção do sulco nasogeniano ao tragus utilizou-se a haste do paquímetro para mensuração de estruturas externas, procedendo da seguinte forma: traçouse uma reta com um lápis dermatográfico marca Tombowâ dando continuidade ao sulco até a altura da mandíbula usando-se uma régua como guia e em seguida fez-se a medida desta projeção até o ponto central do tragus (Figura 1).

Todos os indivíduos foram fotografados antes e após o tratamento, mantendo-se à distância de $50 \mathrm{~cm}$ dos sujeitos com uma câmera digital marca MinoltaâDimagê V. Os participantes dos grupos passaram individualmente pelas etapas de anamnese e avaliação, e de acordo com os resultados destas, foram elaborados protocolos de exercícios faciais especialmente para essa pesquisa baseados em diversos autores ${ }^{12-17}$ direcionados para testa e papada, olhos, bochechas e lábios. Esses protocolos foram aplicados e treinados em grupo semanalmente, com a orientação do fonoaudiólogo, havendo também atendimentos individuais posteriores para manipulação da face. No momento individual (15 minutos), onde se processava a manipulação intra-oral, aproveitava-se para fazer observações mais específicas, inclusive realizavam-se exercícios específicos para cada paciente. Foi entregue aos participantes do grupo um protocolo sobre os cuidados ${ }^{11}$ com a face. Finalmente, foi proposta aos pacientes a realização dos exercícios, diariamente, pelo período de oito semanas. A avaliação, o tratamento e a reavaliação tiveram uma duração total de 16 sessões com um cronograma pré-estabelecido. As manipulações da face ocorreram do sexto ao décimo terceiro encontro.

Para a avaliação da eficácia do programa proposto foi lançado um protocolo de desempenho ${ }^{11}$. Foi questionado sobre: cuidados com a face, freqüência dos exercícios, áreas da face para as quais direcionou os exercícios e modificações percebidas na face após o período de tratamento.

O programa utilizado para a entrada e construção do banco de dados foi o software Officer Excel da Microsoftâ, sendo a análise dos dados realizada através de estudos estatísticos com distribuição tabular e gráfica, utilização do software SPSS 10 for Windows. Os testes realizados foram: teste $T$ de Student para amostras pareadas e o teste não paramétrico de Wilcoxon, quando analisadas as comparações através de escalas. Foi considerado estatisticamente significante para o valor de $p<0,05$.

Este estudo foi analisado e aprovado pelo Comitê de Ética em Pesquisa do Centro de Ciências da Saúde da Universidade Federal de Pernambuco, ofício número 257/2003-CEP/CCS e incluiu o Termo de Consentimento Livre e Esclarecido por escrito de acordo com a Resolução 196/96 - Diretrizes e Normas Regulamentadoras de Pesquisa Envolvendo Seres Humanos do Conselho Nacional de Saúde.

\section{RESULTADOS}

Os resultados referentes à distribuição das idades dos pacientes, ao percentual de cuidados com a face, das áreas da face para onde foram direcionados os exercícios, das áreas da face onde foram percebidas as modificações e das médias das medidas da projeção do sulco nasogeniano ao tragus são expressos nos Tabelas e Figuras que se seguem.

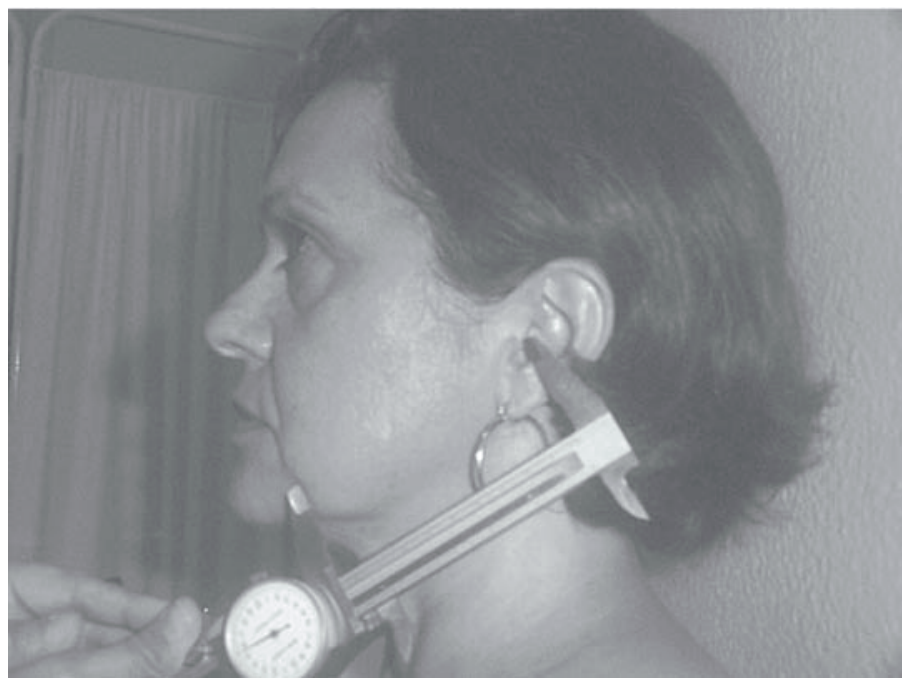

Figura 1 - Mensuração da projeção do sulco nasogeniano ao tragus 
Tabela 1 - Distribuição das idades (em anos) dos pacientes submetidos à intervenção fonoaudiológica

\begin{tabular}{ll}
\hline Iniciais pacientes & Idades \\
\hline Paciente 1 $(\mathrm{A})$ & 38 \\
Paciente 2 $(\mathrm{C})$ & 38 \\
Paciente 3 $(\mathrm{M})$ & 38 \\
Paciente 4(ME) & 41 \\
Paciente 5 $(\mathrm{MJ})$ & 42 \\
Paciente 6 $(\mathrm{MM})$ & 63 \\
Paciente 7 (MR) & 45 \\
Paciente 8 $(\mathrm{N})$ & 33 \\
Paciente 9 $(\mathrm{S})$ & 43 \\
Paciente 10 $(\mathrm{V})$ & 54 \\
\hline
\end{tabular}

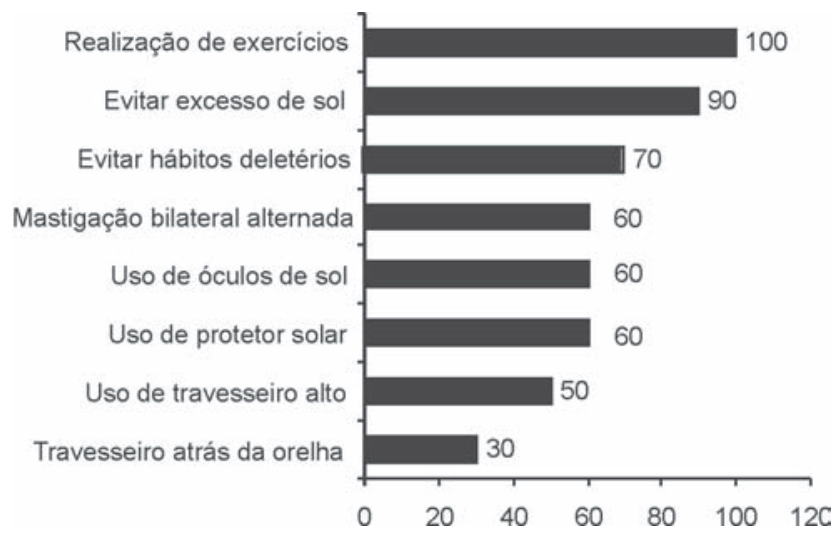

Figura 2 - Distribuição do percentual de cuidados com a face dos pacientes após o tratamento fonoaudiológico

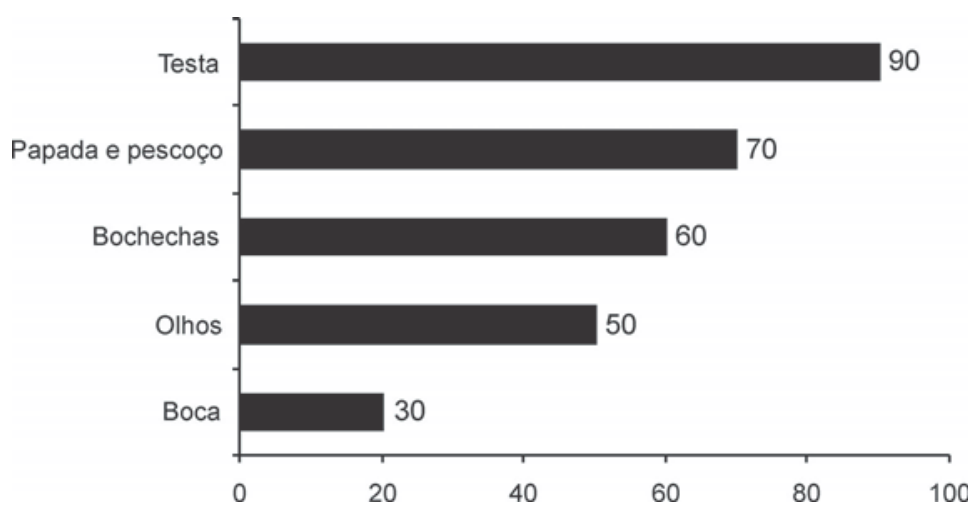

Figura 3 - Distribuição do percentual da área da face para onde foram direcionados os exercícios durante o tratamento fonoaudiológico 
Tabela 2 - Distribuição do percentual da área da face onde foram percebidas modificações

\begin{tabular}{lcr}
\hline & $\mathrm{n}$ & $\%$ \\
\hline Presença de modificação & 10 & 100,0 \\
Sim & 0,0 & 0,0 \\
Não & 07 & 70,0 \\
\hline Área da face modificada & 04 & 40,0 \\
Testa & 06 & 60,0 \\
Olhos & 04 & 40,0 \\
Bochechas & 03 & 30,0 \\
Papada e pescoço & 07 & 70,0 \\
Boca & 03 & 30,0 \\
\hline Percepção da modificação por outros & \\
Sim & 03
\end{tabular}

Tabela 3 - Distribuição do percentual das modificações percebidas na face durante e após o tratamento fonoaudiológico

\begin{tabular}{lc}
\hline Modificações na face & $\%$ \\
\hline Suavidade & 90 \\
Relaxamento & 80 \\
Rugas mais suaves & 80 \\
Firmeza & 40 \\
Leveza & 60 \\
Descansada & 40 \\
Rejuvenescimento & 40 \\
\hline
\end{tabular}

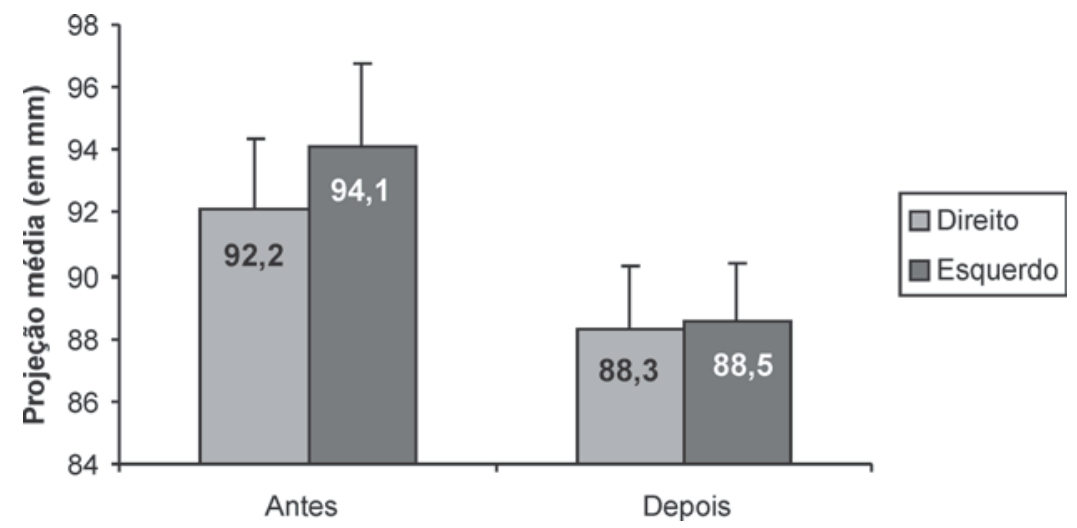

Figura 4 - Média das medidas $(\mathrm{em} \mathrm{mm}$ ) da projeção do sulco nasogeniano ao tragus direito e esquerdo antes e depois da intervenção fonoaudiológica (teste T de Student para amostras pareadas e o teste não paramétrico de Wilcoxon, quando analisadas as comparações através de escalas. Foi considerado estatisticamente significante para o valor de $p<0,05$ )

Em ambos os lados houve uma redução estatisticamente significativa, onde no lado direito a projeção média era de $92,2 \mathrm{~mm}$ e após o tratamento reduziu para $88,3 \mathrm{~mm}(p=0,023)$ e no lado esquerdo a projeção média era de $94,1 \mathrm{~mm}$ e passou para $88,5 \mathrm{~mm}(p=0,001)$. 


\section{DISCUSSÃO}

Observa-se que o perfil desta amostra, em relação ao gênero e idade é semelhante ao perfil de outra pesquisa desenvolvida anteriormente ${ }^{5}$ que realizou experimento com oito voluntários, seis de gênero feminino e dois do gênero masculino, com faixa etária entre 31 e 66 anos. Em relação à atividade profissional do grupo, não foi referido na literatura nenhum trabalho com grupos de professores.

O tempo de duração do tratamento total foi de quatro meses (16 encontros), inclusos os processos de avaliação e reavaliação. As manipulações da face ocorreram do sexto ao décimo terceiro encontro, ou seja, durante oito sessões. Na literatura os tempos de tratamentos propostos variaram de cinco sessões ${ }^{3}$, dez sessões ${ }^{2}$, três meses (12 sessões) ${ }^{1,5}$ a cinco meses (20 sessões) ${ }^{4}$. Como se pode observar, o tempo de tratamento realizado estava contido no intervalo de tempo dos tratamentos propostos na literatura.

Os exercícios propostos foram realizados primeiramente em grupo com a orientação da fonoaudióloga, após o término de cada sessão de grupo, todos os voluntários passavam por uma sessão de manipulação da face individualmente. Os exercícios eram realizados uma vez por dia durante toda semana até o próximo encontro. A literatura refere exercícios para casa ${ }^{5}$ ou realizados individualmente ${ }^{2,4}$.

Este tipo misto de abordagem grupal e individual traz vantagens, principalmente em relação aos benefícios de um trabalho de grupo, como o desenvolvimento de espírito coletivo, aumento da motivação, socialização das experiências e em conseqüência do aprendizado, além de fornecer subsídios para o desenvolvimento da auto-imagem facial. Vale salientar que o grupo manteve todos os dez elementos durante os quatro meses da realização do tratamento, o que demonstra o alto nível de coesão do grupo. No momento individual, onde se processava a manipulação da face, aproveitava-se para fazer observações mais específicas, inclusive realizavam-se exercícios de acordo com a necessidade de cada paciente.

Trabalhou-se durante o tratamento além de orientações para os cuidados da face, exercícios isotônicos como a aeróbica facial ${ }^{14}$, isométricos e isocinéticos, massagens, manipulações faciais ${ }^{12} \mathrm{e}$ equilíbrio funcional estomatognático, incluindo o treino mastigatório.

A idade parece não ter tido influência nos resultados em relação aos trabalhos faciais. Analisando algumas respostas do protocolo de desempenho observa-se que em relação à freqüência dos exercícios (A) e (M) faziam os exercícios em média 3 vezes na semana e, comentaram que esqueciam algumas vezes e não tinham muita disponibilidade. Já (MM), a paciente mais velha do grupo obteve um bom resul- tado em relação à face e a voz. Referiu fazer os exercícios para a face diariamente de manhã e de noite e que algumas pessoas comentaram que estava mais jovem e questionaram se ela tinha se submetido a alguma plástica no rosto.

O protocolo de desempenho mostrou que todos os pacientes realizaram exercícios para a face. Só a paciente (M) respondeu que se expunha ao sol, alegando gostar muito de tomar sol. E dos seis pacientes que incorporaram a mastigação bilateral alternada, só dois apresentavam esse padrão anterior ao tratamento fonoaudiológico, embora com suas adaptações o que reforça a necessidade de realização dos exercícios também com estes dois indivíduos. Este dado é corroborado por outros autores que afirmam que são obtidos como resultados da atuação fonoaudiológica na estética da face o equilíbrio miofuncional dos feixes mastigatórios e faciais e das funções do sistema estomatognático ${ }^{8}$. E ao referir a respeito do novo equilíbrio adquirido pelo paciente em sua forma de mastigar, engolir e repousar a boca após o tratamento fonoaudiológico para a face ${ }^{2}$.

Comparando as áreas da face para as quais os exercícios foram preferencialmente direcionados, com as áreas da face em que os pacientes perceberam um resultado positivo, observa-se que a concordância se deu em ordem decrescente nas áreas das bochechas e boca (100\%), área dos olhos (80\%), área da testa $(70 \%)$ e áreas de papada e pescoço (57\%), Ou seja, os exercícios, que mais se perceberam resultados positivos foram os das áreas das bochechas e da boca.

Todos os pacientes perceberam alguma modificação na face após o tratamento fonoaudiológico. Sete pacientes responderam que tiveram alguma modificação na face percebida por outras pessoas, Os três pacientes que responderam não haver sido percebido modificação por outras pessoas (A), (M) e (V) foram os que realizaram os exercícios com as menores freqüências, inclusive menores que o solicitado pela fonoaudióloga, a paciente $(\mathrm{V})$ realizou os exercícios cinco vezes na semana e as pacientes (A) e (M) três vezes na semana. Desta forma, podese considerar como fundamental a freqüência diária dos exercícios.

A suavidade e o relaxamento da pele estão de acordo com o outro trabalho ${ }^{5}$ que refere tornar-se à face descansada, relaxada e serena após a realização dos exercícios isométricos. Em relação às rugas, oito pacientes observaram que elas tornaramse mais suaves e quatro pacientes referiram ter notado uma maior firmeza em relação ao tônus da face. Achados esses em consonância com este mesmo trabalho 5 em que os pacientes observaram diminuição das rugas, marcas de expressão e flacidez facial, e outro autor ${ }^{2}$ que refere sobre a suavização das marcas de expressão. Relativo ao aspecto geral da 
face, foi referida a sensação de leveza por seis pacientes e descanso e rejuvenescimento por quatro pacientes, estando todos esses relatos de acordo com a pesquisa ${ }^{8}$ que afirma a respeito da melhora do aspecto do tegumento além da harmonia e simetria facial, funcional e estética e com o trabalho ${ }^{2}$ que relata sobre o aspecto mais harmonioso do rosto e a presença de uma expressão mais suave.

Visto que as fotografias não fornecem comprovação científica, esta documentação teve um caráter de observação da evolução e ilustrativo.

Não foi encontrado na literatura nenhum trabalho que referisse a respeito da mensuração da projeção do sulco nasogeniano ao tragus da hemiface direita e esquerda como forma de comparar a situação anterior e posterior ao tratamento fonoaudiológico aplicado à estética facial. Pode-se considerar que este tipo de medida apresenta uma objetividade e um valor científico maior do que apenas a documentação fotográfica.

Avaliando a média da projeção do sulco nasogeniano ao tragus antes e depois da intervenção fonoaudiológica, observou-se que antes do tratamento a diferença entre o lado direito e esquerdo era, em média, de 1,9mm e após o tratamento essa diferença foi de $0,2 \mathrm{~mm}$. Tanto antes do tratamento $(p=0,128)$ quanto depois $(p=0,445)$, não houve dife- rença estatisticamente significativa entre as projeções segundo o lado da face, porém foi notório o equilíbrio após o tratamento. Em ambos os lados houve uma redução estatisticamente significativa, onde no lado direito a projeção média era de $92,2 \mathrm{~mm}$ e após o tratamento reduziu para $88,3 \mathrm{~mm}(p=0,023)$ e no lado esquerdo a projeção média era de $94,1 \mathrm{~mm}$ e passou para $88,5 \mathrm{~mm}(p=0,001)$.

Embora ainda não referida, uma explicação para redução das medidas pode estar associada ao aumento do tônus na musculatura da face, em especial dos músculos bucinadores e elevadores do lábio superior, resultando em uma angulação maior do sulco nasogeniano e consequente diminuição da distância entre a projeção deste sulco ao tragus.

\section{CONCLUSÃO}

Comparando as situações pré e pós-tratamento fonoaudiológico proposto para face: a) Foi constatada uma redução significativa das medidas da projeção do sulco nasogeniano ao tragus em ambos os lados, após o tratamento fonoaudiológico; b) Foi observado um notório equilíbrio entre as medidas da projeção do sulco nasogeniano ao tragus das hemifaces direita e esquerda.

\section{ABSTRACT}

Purpose: to describe the modifications in the face behavior after the speech language pathology treatment proposed for the face, by comparing the measurements of the projection related to the nasolabial sulcus to the tragus of these patients before and after the speech language pathology treatment. Methods: the surveyed population was mad up by 10 teachers treated at the "Hospital dos Servidores do Estado" aged between 33 and 63 years. Data collection process was made up by protocols, anamnesis and evaluation, performance protocol, in addition to the photographic record. A measurement of the nasolabial sulcus to the tragus was carried out. Throughout the 16-session, we carried on specific orientation about face isotonic, isometric and isokinetic exercises, massages, facial manipulation, functional equilibrium of the stomatognathic system besides the orientations about faces care. Results: a significant reduction in the measurements of the nasolabial sulcus projection to tragus in both sides was verified and also a significant balance was observed between the right and left sides. Conclusion: the treatment program proposed for the face reduced the measurements as for the projection the nasolabial sulcus to the tragus and provided balance among such measurements.

KEYWORDS: Speech; Language and Hearing Sciences; Face; Esthetic

\section{REFERÊNCIAS}

1. Silva VLM. Ginástica facial isométrica proprioceptiva. Inform Cons Reg Fonoaudiol $2^{\mathrm{a}}$ região. $2000 ;$ p. 16.

2. Franco MZ. Estética facial: uma nova atuação fonoaudiológica. Inform Cons Reg Fonoaudiol $2^{\mathrm{a}}$ região. 2000; p.15.

3. Franco MZ, Scattone L. Fonoaudiologia e dermatologia um trabalho conjunto e pioneiro na suavização das rugas de expressão facial. Fono Atual. 2002; 22:60-6.

4. Tasca SMT. Programa de aprimoramento muscular em fonoaudiologia estética facial (PAMFEF). Barueri: Pró-fono; 2002. 186 p.

5. Takacs AP, Valdrighi V, Assencio-Ferreira VJ. Fonoaudiologia e estética: unidas a favor da beleza facial. Rev CEFAC. 2002; 4(2):111-6. 
6. Ulson SMA. Estética facial: possibilidades de intervenção fonoaudiológica no diagnóstico e tratamento das rugas de expressão. In: Marchesan IQ, Zorzi J, organizadores. Tópicos em fonoaudiologia 2002/2003. Rio de Janeiro: Revinter; 2003. p. 315-39.

7. Pierotti MS. Fonoaudiologia e estética facial. In: V Congresso Internacional, XI Congresso Brasileiro, I Encontro Cearense de Fonoaudiologia, 2003, Fortaleza. Anais do V Congresso Internacional, XI Congresso Brasileiro, I Encontro Cearense de Fonoaudiologia, Fortaleza, 2003. CD-ROM.

8. Toledo PN. Atuação fonoaudiológica na estética da face. São Paulo: 2003. 4 p.

9. Franco MZ. Fonoaudiologia e estétical: um novo alcance da motricidade oral. In: Comitê de Motricidade Orofacial - SBFa. Motricidade orofacial: como atuam os especialistas. São José dos Campos: Pulso; 2004. p. 289-95.

10. Pierotti S. Atuação fonoaudiológica na estética facial. In: Comitê de Motricidade Orofacial - SBFa. Motricidade orofacial: como atuam os especialistas. São José dos Campos: Pulso; 2004. p. 281-7.

11. Paes MCNM. Estética facial e voz: uma nova interface. [monografia]. Recife (PE): Universidade Federal de Pernambuco; 2004. 67 p.

12. Toledo PN. Curso atuação fonoaudiológica na estética da face. São Paulo, julho, palestra proferida, 2003. 13. Siqueira R. Guia de automassagem e estética facial. 2. ed. São Paulo: Máster Book; 1998. 46 p. 14. Rammage L. Vocalizing with ease: a selfimprovement guide. Vancouver: National Library of Canada; 1996.115 p.

15. Colas M. Juventude e beleza através da ginástica facial. Rio de Janeiro: Enelivros; 1990. 141 p.

16. Petkova M. Ginástica facial isométrica: mantenha a juventude do seu rosto. 4. ed. São Paulo: Agora; $1989.78 \mathrm{p}$.

17. Rowland C. Facial magic. 1997. VHS.

RECEBIDO EM: 16/09/2006

ACEITO EM: 13/04/2007

Endereço para correspondência

R. Isaac Salazar, 45 B, 1202

Recife - PE

CEP: $52.050-160$

Tel: (81) 32676579/ 99755558

E-mail: carolp@elogica.com.br 